\title{
The Opioid Epidemic in the United States: $A$ Call for Practice Change in Pediatric Outpatient Surgeries
}

\author{
Emma Bacharach, MD ${ }^{1}$, Osama AI-Omar, MD, MBA, FACS ${ }^{1}$
}

ABSTRACT

Author affiliations are

listed at the end of this

While narcotic analgesics are effective in treating post-surgical pain, these commonly article. prescribed medications have been shown to carry significant risks of inappropriate use, chronic dependence, and overdose leading to death. The epidemic of opioid use in the USA is multifactorial and in part, is the product of excessive prescribing by surgeons treating postoperative pain. ${ }^{1,2}$ In recent years, there has been great emphasis on limiting postoperative narcotic prescribing in adult patients. More recently, this has extended to limiting narcotic use in pediatric patients as well due to rising concerns of persistent use after surgery and serious adverse events in this population including death. ${ }^{3}$

Correspondence to: Emma Bacharach, MD West Virginia University, WVU Medicine Children's Hospital

emma.bacharach@hsc.wvu.edu

\section{KEYWORDS}

Narcotic, Pediatric, Surgeries, Opioid Epidemic, Pediatric Urology

While narcotic analgesics are effective in treating post-surgical pain, these commonly prescribed medications have been shown to carry significant risks of inappropriate use, chronic dependence, and overdose leading to death. The epidemic of opioid use in the USA is multifactorial and in part, is the product of excessive prescribing by surgeons treating postoperative pain. ${ }^{1,2}$ In recent years, there has been great emphasis on limiting postoperative narcotic prescribing in adult patients. More recently, this has extended to limiting narcotic use in pediatric patients as well due to rising concerns of persistent use after surgery and serious adverse events in this population including death. ${ }^{3} \mathrm{CDC}$ data demonstrated that pediatric opioid-related deaths in the US have increased by $268 \%$ from 1999 to 2016 . Approximately 9,000 children and adolescents died in this seventeen year timeframe from opioids. Of these deaths, $73 \%$ were due to prescription opioids, and $7 \%$ were in children ages 0-4 years old. ${ }^{4}$ Commonly implicated medications have included hydrocodone, oxycodone, morphine, tramadol, codeine and buprenorphine. The FDA released more restrictive warnings in 2017 regarding codeine and tramadol in pediatric patients. Codeine is contraindicated in children under twelve years old and tramadol in children under seventeen years old, due to multiple cases of over-sedation, apnea and death. ${ }^{5}$

Historically post-surgical pain in the pediatric population was generally undertreated. This is largely due to early misconceptions about pediatric pain sensation capabilities. Additionally, infant and young children's inability to verbalize or convey pain presence and severity have led to undertreatment. ${ }^{6}$

Currently, there is concern that narcotic pain medication is overprescribed in the pediatric population. This concern is supported by the adverse effects and events associated with their use. In one study by Chung et al, $15 \%$ of their pediatric population cohort over one year were prescribed and filled one or more opioid prescriptions.7 Notably, of these 1.3 million opioid prescriptions, over $50 \%$ were for outpatient medical and dental procedures. They found that 1 of every 2611 opioid prescriptions resulted in an opioid related ED visit (excluding Gl or skin effects), hospitalization, or death. The vast majority of these adverse events, $89 \%$, were from narcotics directly prescribed to the child, and in $71 \%$, the narcotic medication was taken in a manner consistent with how the medication was originally 
prescribed. ${ }^{7}$

The adverse effects in the adult population have been well established and include sedation, respiratory depression, dizziness, ileus, constipation, opioid induced hyperalgesia/pain sensitivity, immunosuppression, and hormonal dysfunction. ${ }^{8,9}$ Evidence regarding adverse effects specific to opioid use in the pediatric population is more limited, in part because research involving pediatric patients and opioids is limited by ethical issues involved in enrolling pediatric patients for clinical trials. Major adverse effects of opioids well known to the pediatric population include dizziness, sedation, dose-related respiratory and CNS depression leading to apnea and death, constipation, and nausea and vomiting.,10 Accidental ingestion is also an important risk especially for children under six years old. From 2010$2013,13.8 \%$ of the total ED visits for unsupervised medication exposures in the US were due to oral narcotic ingestions. ${ }^{11}$ Additional risks in prescribing these medications to pediatric patients include inappropriate use by family members or others in the home and physical dependence and addiction. Increased availability of these narcotics in the home and community has been shown to contribute to overdoses in all age groups. ${ }^{12}$

Interestingly, comparisons between pain control using narcotic and non-narcotic medications postoperatively have demonstrated similar efficacy in treating pain in adults as evidenced by similar reported pain scores. ${ }^{9}$ Given the lower risks associated with non-narcotic pain medication use and the similar efficacy of non-narcotics in pain control, the use of narcotics in the pediatric population should be limited. Narcotics should be considered a second line treatment and avoided, if possible, especially for outpatient surgeries.

At WVU Medicine Children's, we implemented a new strategy since May 2019 of treating postoperative pain without use of any narcotics in pediatric urology patients undergoing outpatient same day surgery that includes open and laparoscopic/ robotic procedures. Our protocol for perioperative pain management starts during the surgery with adequate local anesthesia for the incisional site with bupivacaine (Marcaine) $0.25 \%$ or caudal anesthesia.
This is followed by post-operative scheduled Tylenol and Motrin, alternating for the next twenty four hours, and then as needed.

We reviewed our data for all outpatient procedures from May 1, 2019 until March 1, 2020. Of the 330 cases treated without narcotics, we have observed no emergency department visits and have received only two parental complaints of poorly managed pain. One of those two parents stated that her son's pain after penile surgery was inadequately managed with non-narcotic medication, but she preferred not to give him a narcotic one.

Overall, our experience at WVU suggests that it is feasible to eliminate postoperative narcotic use in children undergoing outpatient surgery. This can be done without compromising pain control or patientfamily satisfaction. Increased community awareness of risks associated with opioids is growing and will likely influence the preference of postoperative analgesia toward no narcotics. A large-scale implementation of this practice would assist in decreasing the morbidity and mortality associated with the current US opioid epidemic.

\section{AUTHOR AFFILIATIONS}

1. West Virginia University, WVU Medicine Children's Hospital, Morgantown, West Virginia

\section{REFERENCES}

1. Theisen K, Jacobs B, Macleod L, Davies B. The United States opioid epidemic: a review of the surgeon's contribution to it and health policy initiatives. BJU Int. 2018;122(5):754-759.

2. Hah JM, Bateman BT, Ratliff J, Curtin C, Sun E. Chronic Opioid Use After Surgery: Implications for Perioperative Management in the Face of the Opioid Epidemic. Anesth Analg. 2017; 125(5):1733-1740.

3. Harbaugh CM, Lee JS, Hu HM, et al. Persistent Opioid Use Among Pediatric Patients After Surgery. Pediatrics. 2018;141(1):e20172439.

4. Gaither J, Shabanova V, Leventhal J. US national trends in pediatric deaths from prescription 
and illicit opioids, 1999-2016. Jama Netw Open.

2018;1.

5. Fortenberry M, Crowder J, So TY. The use of codeine and tramadol in the pediatric population-what is the verdict now? Journal of Pediatric Health Care : Official Publication of National Association of Pediatric Nurse Associates \& Practitioners. 2019 Jan;33(1):117123.

6. Finley GA, Chorney J, Campbell L. Not small adults: the emerging role of pediatric pain services. Can J Anesth. 2014;61(2):180-187.

7. Chung CP, Callahan ST, Cooper WO, Dupont WD, Murray KT, Franklin AD, Hall K, Dudley JA, Stein CM, Ray WA. Outpatient opioid prescriptions for children and opioid-related adverse events. Pediatrics. 2018;142(142).

8. Benyamin R, Trescot AM, Datta S, et al. Opioid complications and side effects. Pain Physician. 2008;11(2 Suppl):S105-S120.

9. Martinez L, Ekman E, Nakhla N. Perioperative opioid-sparing strategies: utility of conventional NSAIDs in adults. Clin Ther. 2019;41:2612-2628.

10. Geib AJ, Babu K, Ewald MB, Boyer EW. Adverse effects in children after unintentional buprenorphine exposure. Pediatrics. 2006;118:1746-1751.

11. Lovegrove MC, Weidle NJ, Budnitz DS. Trends in emergency department visits for unsupervised pediatric medication exposures, 2004-2013. Pediatrics. 2015;136(4):e821-e829.

12. Harbaugh CM, Gadepalli SK. Pediatric postoperative opioid prescribing and the opioid crisis. Curr Opin Pediatr. 2019;31(3):378-385. 